\title{
ADDITIONAL ENERGY LOSSES FROM ASYMMETRIC AND NON-SINUSOIDAL CURRENT IN AN ELECTRICAL FACILITY AND METHODS OF THEIR REDUCTION
}

\author{
Evgeniy V. Tarasov ${ }^{1 a}$, Leonid L. Bulyga ${ }^{1}$, Vasily Ya. Ushakov ${ }^{1}$, Nikolay N. Kharlov ${ }^{1}$ \\ ${ }^{1}$ National Research Tomsk Polytechnic University, 634050 Tomsk, Russia
}

\begin{abstract}
Influence of the asymmetry and higher harmonics of current on the operation of an electrical facility is analyzed. The level of additional losses from the asymmetric and non-sinusoidal currents is evaluated for a $110 \mathrm{kV}$ electrical network in the Siberian Region of the Russian Federation. Methods for reducing the additional energy losses in the electrical facility are suggested.
\end{abstract}

\section{Introduction}

Electrical power losses are an important factor characterizing the efficiency of electrical networks. To estimate them, accurate calculations of losses are required taking into account the maximal number of external factors.

To calculate tariffs for electricity, only technological power losses are taken into account. Nowadays, additional energy losses are not included in calculations of energy losses by the method introduced by Order No. 326 of the Ministry of Energy of the Russian Federation, because they refer to commercial losses and are not included into the bill of fare. The additional losses are taken to mean the power (electricity) losses from the non-sinusoidal and asymmetric current in an electrical facility. The lack of normative and methodological documents for calculation of the additional losses does not allow the total losses to be estimated objectively, which leads to economic losses of power supply companies from underestimation of energy.

\section{Additional energy losses in electrical facilities}

In electric motors additional losses from non-sinusoidal and asymmetric current lead to an increase of the total temperature of the machine and local overheating, most probably in a rotor. This causes additional heating of stator and rotor leading to accelerated aging of insulation and reduced efficiency factor of motors.

In transformers high voltage and current harmonics cause an increase in the hysteresis losses and losses on eddy currents in steel as well as in ohmic losses in windings. The service time of insulation is also reduced.

\footnotetext{
${ }^{\mathrm{a}}$ Corresponding author : e.tarasov@inbox.ru
} 
In electrical transmission line (ETL) additional active power losses are also due to non-sinusoidal and asymmetric currents and voltages. In an overhead line this reduces the efficiency of electricity transmission, and in cable lines this also leads to accelerated thermal aging of insulation due to dielectric losses and increased heating of cable sheaths due to skin and proximity effects.

Additional losses are especially dangerous for electrical capacitors, because they lead to their overheating. For asymmetric voltage, capacitor banks are nonuniformly loaded by reactive power of phases, which makes impossible the complete utilization of the capacitor bank. [1].

\section{Evaluation of additional losses in a $110 \mathrm{kV}$ power transmission line}

The authors have data on the parameters of the ETL operation obtained during instrumental examinations, including the data from "Altai Energo."

These examinations were carried out using AR-5 portable network analyzers to measure the amount and quality of the electric energy.

The device is registered in the Register of Measuring Instruments approved for application in the territory of the Russian Federation. The parameters of the network operation were fixed at predetermined time intervals.

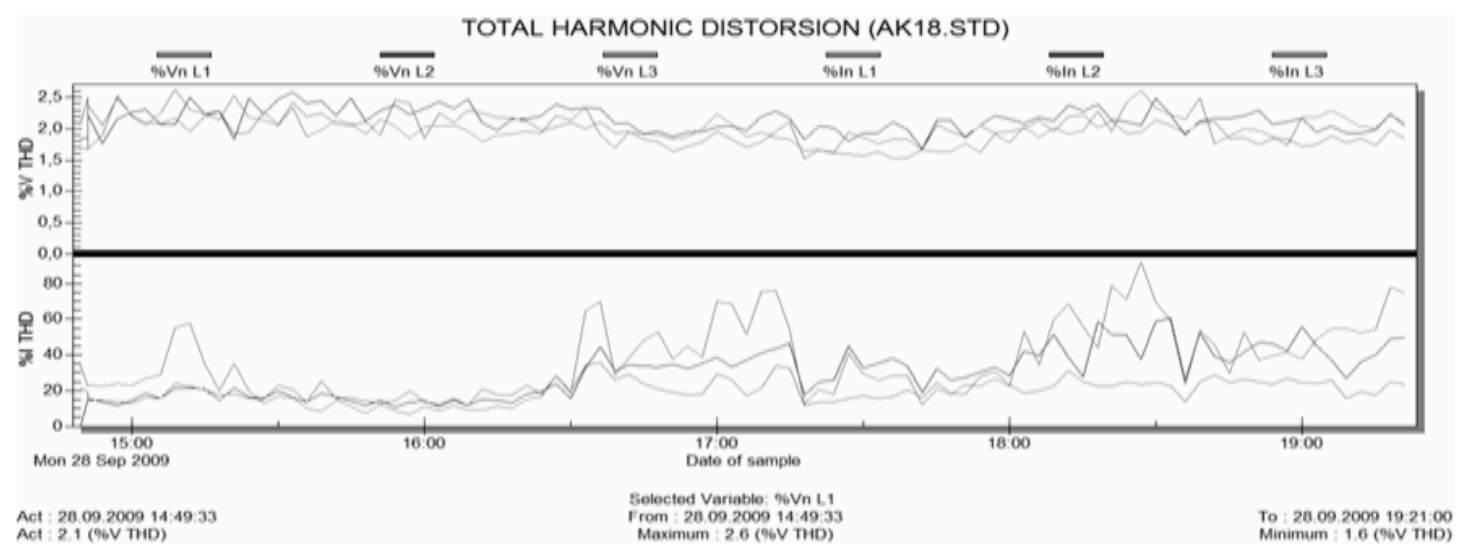

Figure 1. Plot of the total harmonic distortions of the voltage on a $110 \mathrm{kV}$ bus substation (at the top) and of the current in the AK-18 ETL (at the bottom)

The plots refer to the ETL connecting the "Arbuzovskaya" and "Korchynskaya" substations. The ETL utilizes an AC-150 wire and has the length $\mathrm{L}=23 \mathrm{~km}$. Significant distortions of the current waveform can be seen, namely, the total harmonic distortion reaches $80 \%$.

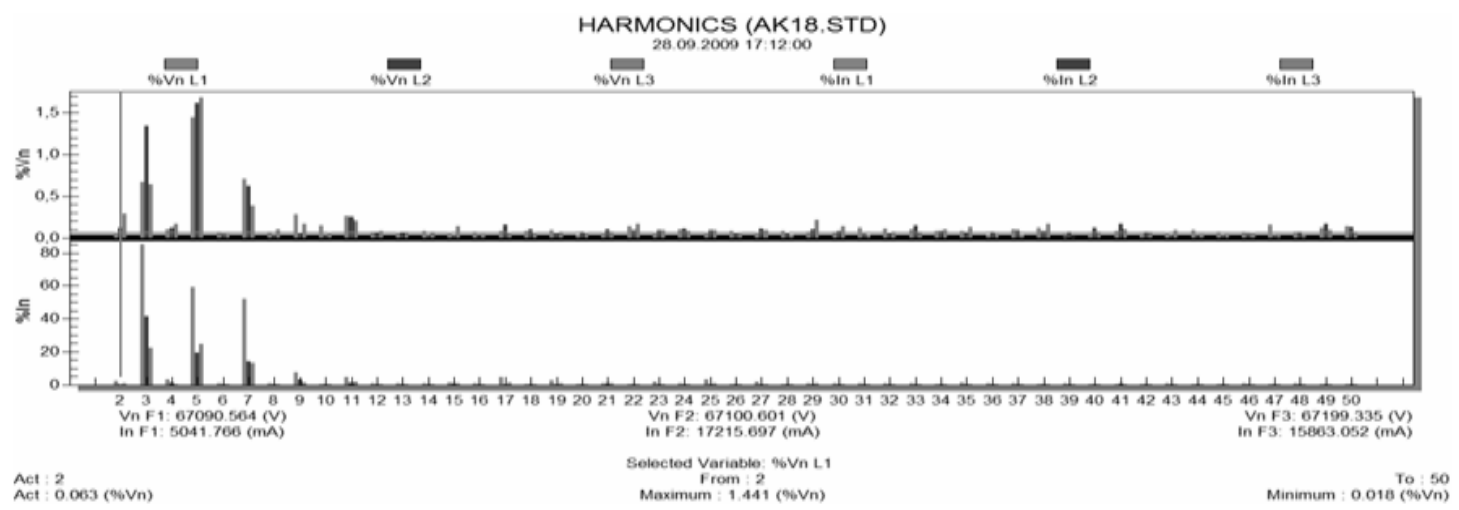


Figure 2. Histograms of harmonic voltage components on the $110 \mathrm{kV}$ bus substation (at the top) and of current for the AK-18 ETL (at the bottom) at one of the moments of maximal harmonic current distortion

Fig. 2 shows the spectral composition of the voltage and current. Characteristically, it is wideband and is determined mainly by the harmonic components with relative frequencies of 3,5 , and 7 . The third harmonic current component is $80 \%$, the fifth is $60 \%$, and the seventh is $50 \%$.

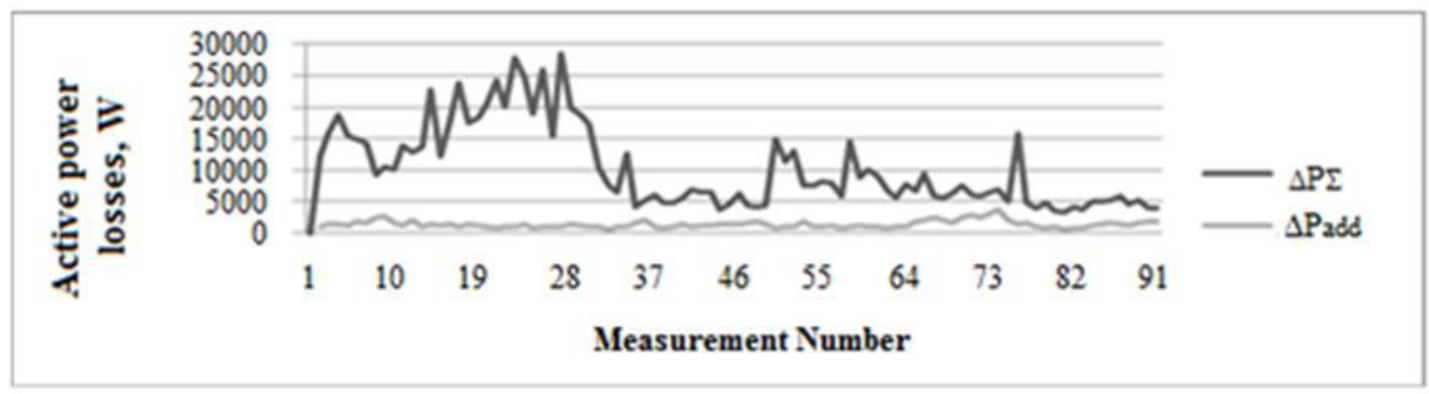

Figure. 3. Structure of the active power losses in the $110 \mathrm{kV}$ ETL (from substation "Arbuzovskaya" to substation "Korchynskaya")

Fig. 3 shows the structure of the active power losses calculated by the program Calculation of Additional Losses in Single and Double Electric Transmission Lines taking into account the additional losses from the asymmetric and non-sinusoidal current. The average value of the active power losses in the ETL per day was $10.4 \mathrm{~kW}$. The average additional losses of the active power per day were $1.4 \mathrm{~kW}$. Fig. 4 shows the relative fraction of the additional losses in the ETL as a percentage of the total losses. The average value was $13.2 \%$.

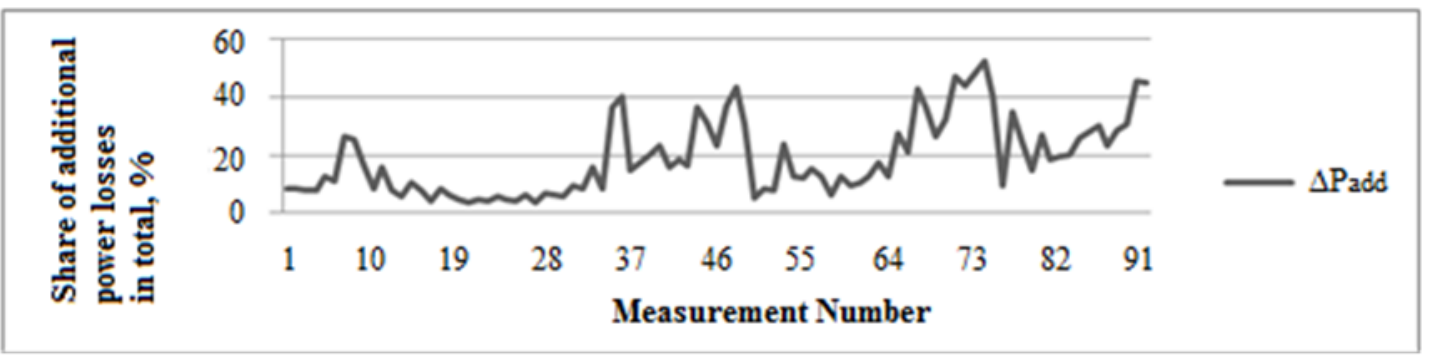

Figure. 4. Relative fraction of the additional active power losses from the total power losses

\section{Methods of reduction of the additional losses of electricity}

To reduce the additional energy losses in an electrical facility, 3 main methods are used:

1. Schematic solutions: connection of nonlinear loads to a separate bus system; distribution of these loads over different units of the power supply system; grouping of converters based on the phase multiplication scheme; connection of the load to a higher-power system.

2. Use of special equipment characterized by a low level of generation of higher harmonics: "unsaturated" transformers and multiphase converters with improved energy performance.

3. Use of filters: connection of narrowband resonant filters in parallel to the load; connection of a filter compensating device; using filter balancing system and active filters connected in series and in parallel. 


\section{Conclusions}

In the course of this work we have elucidated that additional active power and energy losses arise at significant distortions of sinusoidal current and asymmetric regime of operation of electrical equipment. Percentage of these losses makes significant relative fraction of the basic losses, but according to [5], these losses are not taken into account in the calculation of the technological energy losses. Thus, our calculations demonstrated that in the examined $110 \mathrm{kV} \mathrm{ETL}$, the relative fraction of the additional energy losses from non-sinusoidal and asymmetric current reached $13.2 \%$. In this regard, we can make the following conclusions:

1. Additional active power and energy losses must be calculated separately for each line or electrical facility. Calculations should be based on the results obtained by instrumental examination of the operational parameters for the electrical facility.

2. Actual technological losses from non-sinusoidal and asymmetric modes are actually much larger than the power losses calculated based on the instruction stated in [5].

\section{References}

1. J. Arrillaga, N. R. Watson, Power system harmonics. Second edition (University of Canterbury, Christchurch, New Zealand, 2003)

2. R. Sasdelli, G.D. Gobbo, G. Iuculano, IEEE Trans. Instrum. Meas., 49 (2), 460 (2000)

3. J.W. Fourie, J. E. Calmeyer, Proc. 7th Conference in Africa «Africon», 2, 667 (2004)

4. Order No. 326 of the Ministry of Energy of the Russian Federation of December 30, 2008 on the Organization of Works on the Approval of Standards for the Electrical Energy Losses during Energy Transmission over Electrical Transmission Lines. 